\title{
Behavioral and neural correlates of disrupted orienting attention in posttraumatic stress disorder
}

\author{
Stefanie Russman Block ${ }^{1,2,3}$ • Anthony P. $\operatorname{King}^{1} \cdot \operatorname{Rebecca}^{1}$. Sripada ${ }^{1,3}$. \\ Daniel H. Weissman ${ }^{2}$. Robert Welsh ${ }^{1,4}$ • Israel Liberzon ${ }^{1,2,3}$
}

Published online: 13 December 2016

(C) Psychonomic Society, Inc. 2016

\begin{abstract}
Prior work has revealed that posttraumatic stress disorder (PTSD) is associated with altered (a) attentional performance and (b) resting-state functional connectivity (rsFC) in brain networks linked to attention. Here, we sought to characterize and link these behavioral and brain-based alterations in the context of Posner and Peterson's tripartite model of attention. Male military veterans with $\operatorname{PTSD}(N=49$; all deployed to Iraq or Afghanistan) and healthy age-and-gendermatched community controls $(N=26)$ completed the Attention Network Task. A subset of these individuals (36 PTSD and 21 controls) also underwent functional magnetic resonance imaging (fMRI) to assess rsFC. The behavioral measures revealed that the PTSD group was impaired at disengaging spatial attention, relative to the control group. FMRI measures further revealed that, relative to the control group, the PTSD group exhibited greater rsFC between the salience network and (a) the default mode network, (b) the dorsal attention network, and (c) the ventral attention network. Moreover, problems with disengaging spatial attention increased the rsFC between the networks above in the control
\end{abstract}

Electronic supplementary material The online version of this article (doi:10.3758/s13415-016-0488-2) contains supplementary material, which is available to authorized users.

Stefanie Russman Block

srblo@umich.edu

1 Department of Psychiatry, University of Michigan, Ann Arbor, MI, USA

2 Department of Psychology, University of Michigan, 2269 East Hall, 530 Church Street, Ann Arbor, MI 48109, USA

3 VA Ann Arbor Health Care System, Ann Arbor, MI, USA

4 Department of Psychiatry, University of Utah, Salt Lake City, UT, USA group, but not in the PTSD group. The present findings link PTSD to both altered orienting of spatial attention and altered relationships between spatial orienting and functional connectivity involving the salience network. Interventions that target orienting and disengaging spatial attention may be a new avenue for PTSD research.

Keywords Resting-state $\cdot$ Connectivity · fMRI · PTSD · Attention · Orienting

Posttraumatic stress disorder (PTSD) is associated with altered attention in multiple domains. For example, PTSD is often associated with altered attentional biases toward threat (Pineles, Shipherd, Mostoufia, Abramovitza, \& Yovelc, 2009), attentional deficits on neuropsychological tests (Aupperle, Melrose, Stein, \& Paulus, 2012; Polak, Witteveen, Reitsma, \& Olff, 2012; Qureshi et al., 2011), and attention deficit/hyperactivity disorder (ADHD; Spencer et al., 2016). Nonetheless, prior research has not characterized which specific types of attention are impaired in PTSD or the underlying neural mechanisms. Understanding which aspects of attention are affected in PTSD could improve our understanding of the mechanisms by which these deficits influence symptoms. This, in turn, could lead to new treatments that target these processes.

Experimental research has identified three important components of attention: alerting, orienting and conflict monitoring (Posner \& Petersen, 1990). The alerting component maintains vigilance for novel or unexpected stimuli, the orienting component shifts attention from one item to another and limits focus to a subset of inputs, and the conflict monitoring component signals the activation of competing response tendencies. These three components of attention can be isolated 
using the Attention Network Task (ANT; Fan, McCandliss, Sommer, Raz, \& Posner, 2002).

To date, only two behavioral studies have examined the ANT in PTSD, one reporting deficits in conflict monitoring (Leskin \& White, 2007), and the other reporting deficits in orienting attention (Barlow-Ogden \& Poynter, 2012). Other cognitive tests such as the Stroop task, the trail-making test, and the continuous performance test also suggest deficits in orienting and conflict monitoring, although the results are mixed (De Bellis, Woolley, \& Hooper, 2013; Eren-Koçak, Kılıç, Aydın, \& Hızlı, 2009; Jenkins, Langlais, Delis, \& Cohen, 2000; Johnsen \& Asbjørnsen, 2008; LaGarde, Doyon, \& Brunet, 2010; Polak et al., 2012; Shucard, McCabe, \& Szymanski, 2008; Steudte-Schmiedgen et al., 2014; Vasterling et al., 2002). Because some neuropsychological tests do not effectively isolate these components of attention from other executive functions or from each other (Brock \& Clinton, 2007; Perry \& Hodges, 1999) interpreting their findings as indicating conflict monitoring and orienting attention deficits is challenging. Additionally, many such studies do not integrate clinical findings with the functional neuroanatomy of attention (Qureshi et al., 2011; Weierich, Treat, \& Hollingworth, 2008). As a result, there is a gap in our knowledge regarding the neuroanatomical bases of the attentional deficits in PTSD.

Of importance in this regard, neuroimaging studies of the ANT confirm that each component - alerting, orienting, and conflict monitoring - is associated with a distinct set of brain regions. Thus, this task could provide an important tool for determining the neuroanatomical bases of attentional deficits in PTSD. Further along these lines, the brain regions that have been linked to each of the three attentional components in the ANT can be separated into distinct intrinsic connectivity networks (ICNs): groups of brain regions whose low-frequency spontaneous BOLD oscillations fluctuate together at rest (Raichle, 2011; Yeo et al., 2011). For example, the ventral attention network (VAN) is thought to be involved in alerting, the dorsal attention network (DAN) in orienting, and the salience network (SN) in conflict monitoring (Cao et al., 2008; Fan, McCandliss, Fossella, Flombaum, \& Posner, 2005; Muto et al., 2012; Thiel, Zilles, \& Fink, 2004; Westlye, Grydeland, Walhovd, \& Fjell, 2011). ICNs are most readily identified at rest, when there is no task-related activity to "obscure" slow wave oscillations, or complex task instructions (Fox \& Greicius, 2010). ICNs are nonetheless thought to be informative about how functional connections between different brain regions contribute to task performance (Mennes, Kelly, Zuo, \& Martino, 2010).

Because ICNs can be effectively probed using resting-state functional connectivity ( $\mathrm{rsFC}$ ), the number of investigations on rsFC in PTSD has recently grown. These studies have implicated alterations in the mainly the salience network (Gong et al., 2014; Kennis, Rademaker, van Rooij, Kahn, \& Geuze, 2015; Kennis, van Rooij, van den Heuvel, Kahn, \& Geuze, 2016; Nicholson et al., 2016; Sripada et al., 2012a; b; Tursich et al., 2015; Yin et al., 2012; Zhang, Liu, Chen, Li \& Duan, 2015; Zhang, Xie,
Chen, Li \& Liu, 2016; Zhou et al., 2012). Composed of the $\mathrm{dACC}$, insula, and amygdala, the SN is involved in detecting salient stimuli and in switching from a state of rest to a state of task performance (Seeley et al., 2007). Regions of the SN are also thought to be involved in attentional control during task performance (Seeley et al., 2007). For example, it has been suggested that the dACC performs conflict monitoring, which modulates attention to goal-relevant stimuli (Botvinick, Cohen, \& Carter, 2004).

Some recent investigations have also reported PTSDrelated alterations in the ventral (Kennis et al., 2016; Yin et al., 2012; Zhang et al., 2016), and dorsal attention networks (Gong et al., 2014; Kennis et al., 2016; Zhang et al., 2016). The VAN is a closely related, and some believe, partially overlapping network with the SN (Kucyi, Hodaie, \& Davis, 2012), comprised of the right ventral lateral prefrontal cortex (just anterior to the insula of the $\mathrm{SN}$ ), and the temporal parietal junction (TPJ; Sylvester et al., 2012). It is also activated when behaviorally relevant stimuli occur unexpectedly (Vossel, Weidner, Driver, Friston, \& Fink, 2012). The DAN is comprised of the bilateral posterior dorsal lateral prefrontal cortex, the frontal eye fields and the posterior parietal lobe (Corbetta \& Shulman, 2002). It is involved in the voluntary orientation of spatial attention (Vossel et al., 2012). Such PTSD findings raise the possibility that abnormal functioning of attentionrelated ICNs contributes to attentional impairments in PTSD. The relationship between altered ICN functioning and attention impairments, however, remains unclear.

The goal of the present study was to begin to fill this knowledge gap by addressing two aims. First, we sought to determine which aspects of attention are altered in veterans with PTSD as compared to community controls. Second, we sought to determine whether relationships between behavioral measures of attention and resting-state functional connectivity in attention-related ICNs differed between PTSD patients and community controls.

\section{Materials and method}

\section{Participants}

The study was approved by the Institutional Review Boards at the University of Michigan and the Ann Arbor VA. We obtained written informed consent after providing a complete description of the study to participants.

We recruited male combat (Iraq or Afghanistan) veterans with PTSD $(N=49)$ seeking treatment at the Ann Arbor VA (Table 1). Thirteen individuals were ineligible for scanning, or were lost to follow up, leaving 36 to participate in the neuroimaging component of the study. Current PTSD diagnosis was based on DSM-IV criteria, as assessed by the Clinician Administered PTSD Scale (CAPS) using the frequency + 
Table 1 Demographic and clinical characteristics of participants

\begin{tabular}{|c|c|c|c|c|}
\hline Characteristic & $\operatorname{PTSD}(N=49)$ & Control $(N=26)$ & $t / \chi^{2}$ & $p$ \\
\hline Age, $M(S D)$ & $31.49(8.11)$ & $31.00(8.36)$ & 0.246 & .806 \\
\hline Race, $N(\%)$ & & & 7.979 & .092 \\
\hline White/European & $45(92)$ & $20(77)$ & & \\
\hline African American/Black & $4(8)$ & $2(7.7)$ & & \\
\hline Asian American & 0 & $2(7.7)$ & & \\
\hline Biracial & 0 & $2(7.7)$ & & \\
\hline Education, $N(\%)$ & & & 10.958 & .004 \\
\hline Some graduate school or graduate degree & $3(6.1)$ & $9(34.6)$ & & \\
\hline Some college or college degree & $36(73.5)$ & $15(57.7)$ & & \\
\hline High school graduate & $10(20.4)$ & $2(7.7)$ & & \\
\hline CAPS, $M(S D)$ & $77(17.81)$ & N/A & N/A & $\mathrm{N} / \mathrm{A}$ \\
\hline Comorbidities, $N(\%)$ & & N/A & N/A & $\mathrm{N} / \mathrm{A}$ \\
\hline Depression & $26(53)$ & & & \\
\hline Dysthymia & $7(14)$ & & & \\
\hline Bipolar II & $2(4)$ & & & \\
\hline Panic disorder & $4(8)$ & & & \\
\hline OCD & $1(2)$ & & & \\
\hline Social phobia & $3(6)$ & & & \\
\hline Alcohol abuse & $4(8)$ & & & \\
\hline $\mathrm{ADHD}$ & $1(2)$ & & & \\
\hline Medicated $N(\%)$ & $38(77.6)$ & N/A & N/A & $\mathrm{N} / \mathrm{A}$ \\
\hline $\operatorname{mTBI} N(\%)$ & $18(36.7)$ & N/A & N/A & N/A \\
\hline
\end{tabular}

Abbreviations: ADHD, attention-deficit/hyperactivity disorder; mTBI, mild-moderate traumatic brain injury; OCD, obsessive-compulsive disorder; PTSD, posttraumatic stress disorder. Those with mTBI $(M=83.33)$ had greater CAPS scores than those without $\mathrm{mTBI}(M=73.57)$ at a trend level, $t(46)=1.838, p=.072$. No significant differences on any ANT measures were observable between those who were on medication and those who were not. No participants met criteria for serious mental illness such as psychosis, substance dependence or mania. Removal of those with alcohol abuse, bipolar II and ADHD did not affect the results.

intensity $\geq 4$ scoring criteria (Blake et al., 1995). Comorbidity was assessed using the Mini-International Neuropsychiatric Interview (MINI; Sheehan et al., 1998). Those with psychosis, Bipolar I, personality disorders, or suicidal risk were excluded. Participants on psychiatric medications were required to be on a stable dosage for four weeks prior to the fMRI scan. TBI status was established on the basis of the computerized patient record system with a formal consultation at dedicated TBI clinics, or service connection for TBI/postconcussive syndrome.

We recruited age-matched male community controls $(N=$ 26) via flyers, Craigslist, and the University of Michigan clinical studies registry. All controls were free of any Axis I diagnosis (CAPS $\leq 20$, and negative MINI) and psychiatric medications. Individuals with a lifetime history of psychosis or bipolar disorder were excluded. Five individuals did not return for the fMRI portion. Thus, only 21 controls underwent neuroimaging. Since the controls were community volunteers, no medical charts for them were available, and TBI status could not be assessed.
We were adequately powered to detect group differences on the ANT. On the basis of previously reported effect sizes (Leskin \& White, 2007), a sample size of 18 people per group would give $80 \%$ power to detect group differences at an alpha level of 0.05 . The sample sizes of both of our groups were larger than 18.

There were no differences in age, gender, race, education level, mTBI status, or medication use between those who did and those who did not return for the follow-up ( $p$ s $>.1$ in all cases). As often happens (Fischer, Dornelas, \& Goethe, 2001), the groups were significantly different in PTSD symptom severity, with those lost to follow up in the PTSD group reporting higher levels of PTSD symptoms $(M=89.77, S D$ $=14.56)$ than did those not lost to follow up $(M=72.57, S D=$ 14.44), $t(47)=3.164, p<.01$.

\section{Measures}

Attention network task The ANT (Fan et al., 2002) is a combination of the Posner cueing task (Posner, 1980) and 
the Eriksen flanker task (Eriksen \& Eriksen, 1974) that probes three components of Posner and Petersen's (1990) model of attention: alerting, orienting, and conflict monitoring. Each trial contains a row of five arrows, which is preceded by (1) a spatial orienting cue that reveals the likely location of the upcoming target, (2) a non-spatial alerting cue that indicates the target is about to appear (but not its likely location), or (3) no cue (Fig. 1). The participant's task is to determine the direction in which the central target arrow points. Finally, the target arrow is flanked by two arrows on each side, which point in the same direction as the central arrow (congruent trials) or in the opposite direction (incongruent trials).

MRI scanning Participants underwent structural and functional MRI scanning within two weeks after completing the ANT. The fMRI session included an 8 minute resting-state scan followed by separate emotion regulation tasks (King et al., 2016). During the resting-state scan, a white fixation cross on a black background was displayed at the center of the screen. Participants were told to relax, let their minds wander, and keep their eyes fixated on the cross.

MRI data acquisition MRI data was acquired using a Philips 3-T MRI scanner (Phillips Medical Systems, Andover, Massachusetts) at the Ann Arbor VA. We acquired 240 T2*weighted echo planar gradient-recall echo volumes (echo time $=30 \mathrm{~ms}$, repetition time $=2,000 \mathrm{~ms}, 64 \times 64$ matrix, flip angle $=90 \mathrm{deg}$, field of view $=22 \mathrm{~cm}, 42$ contiguous $3-\mathrm{mm}$ axial slices per volume). Five additional volumes were discarded at the beginning of each run to allow for equilibration of the MRI signal. We also obtained a high-resolution T1-weighted structural image (3-D turbo-fast-field-echo, 1-mm isotropic voxel, $256^{2}$ matrix, 180 slices, repetition time $=9.8 \mathrm{~ms}$, echo time $=$ $4.6 \mathrm{~ms}$, flip angle $=8 \mathrm{deg}$ ), to provide for more precise anatomical localization.

\section{Data analyses}

Demographics and behavioral data T-tests and chi-square tests were used to assess group differences in age and education level. Attention components across groups were compared using repeated-measures analyses of covariance (ANCOVAs) controlling for education level. The alerting effect was calculated as mean reaction time (RT) in no cue trials minus mean RT in double cue trials (Fan et al., 2002). The orienting effect was calculated as mean RT in central cue trials minus mean RT in spatial cue trials. The conflict effect was calculated as mean RT in incongruent trials minus mean RT in congruent trials. We also examined the effects of mTBI and medication status on ANT effects within the PTSD group. Finally, we used an ANCOVA to test for group differences in accuracy, controlling for education level.

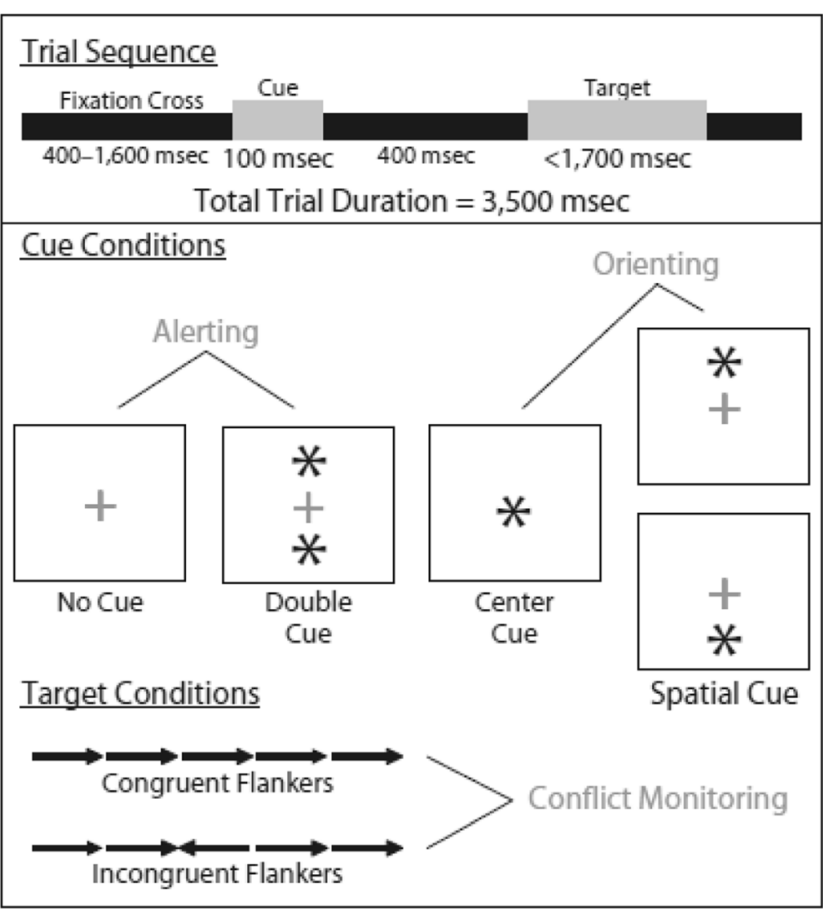

Fig. 1 Schematic of the Attention Network Test, adapted with permission from that described by Jha, Krompinger, and Baime (2007).

MRI data analyses MRI data were analyzed using the statistical parametric mapping software package, SPM8 (Wellcome Department of Cognitive Neurology, London, UK). Functional slices within each volume were sync-interpolated, weighted in time, slice-by-slice, to correct for the staggered sequence of slice acquisition. The functional volumes were then realigned to correct for head motion, spatially normalized to a standard template based on the Montreal Neurological Institute (MNI) reference brain, and smoothed using an 8mm FWHM Gaussian kernel.

To control for nonneuronal noise sources due to heart beat, respiration, and motion (Lund, Madsen, Sidaros, Luo, \& Nichols, 2006), we first extracted the average BOLD time series from structural MRI-derived white matter and cerebrospinal fluid masks. A PCA was performed and the top five components of the time series were added to the model as nuisance covariates. Motion parameters and their first derivatives were also used as nuisance covariates. To control for such micro-movements (Fair et al., 2012) we performed motion scrubbing (removal of volume) using a frame-wise displacement lever arm of $50 \mathrm{~mm}$, a frame-wise threshold of 0.5 , and a scrub window of zero frames before and after the target frame (Power, Barnes, Snyder, Schlaggar, \& Petersen, 2012; Satterthwaite et al., 2012). Finally, we included an overscrubbing threshold of $60 \%$ (Fair et al., 2012). Motion parameters (maximum and mean frame-wise displacement) for the two groups were compared via independent-samples $t$ tests. No global-signal regression was performed to avoid spurious 
anti-correlations (Anderson et al., 2011), but the data were band-pass filtered in the 0.01 to $0.10-\mathrm{Hz}$ band range (Fox et al., 2005).

\section{Attention network task $x$ resting-state functional connec-} tivity analyses We limited ANT $\times$ intrinsic connectivity network analyses to ANT effects that were significantly different between the groups. Prior research has linked the alerting, orienting, and conflict monitoring effects to the VAN, DAN and SN, respectively (Petersen \& Posner, 2012). We thus examined the interaction among each attentional component, the respective ICN connectivity (using an ICN seed), and group status, using a generalized linear model in SPM (e.g., Orienting Effect $\times$ DAN Seed Connectivity $\times$ Group Status). In other words, we examined the link between each ANT effect and resting-state functional connectivity in the associated ICN and then assessed group differences in the strength of this link. Seed coordinates were based on De Luca, Beckmann, De Stefano, Matthews, and Smith (2006), because this network parcellation includes coordinates for all ICNs of interest (Table 2). Findings were small volume corrected within an ICN search mask (De Luca et al., 2006) using a family-wise error (FWE) correction at the cluster level with a voxelwise threshold of $p=.005$ uncorrected. Finally, we repeated ANT $\times$ ICN analyses excluding those in the PTSD group who had mTBIs to determine if the observed findings were influenced by mTBI.

To assess the potential contributions of aberrant SN connectivity reported previously in PTSD to attentional deficits in our study, we examined the association between dACC resting-state functional connectivity, ANT scores, and group status using a generalized linear model in SPM for any ANT effect in which behavioral group differences had been observed (e.g., the association with of dACC resting-state functional connectivity and orienting and/or alerting). The results were thresholded voxel-wise at $p=.005$ uncorrected and then, subsequently, at $p<.05$ (FWE) at the whole brain cluster level. Ten millimeter spheres around significant interaction peaks were extracted to determine the directionality of the association within each group (connectivity was averaged across all voxels within each sphere). Within the PTSD group, we calculated Bonferroni-corrected Pearson correlations between CAPS subscale scores (intrusive, hyperarousal, and avoidance symptoms) and (a) significant interaction peaks and (b) ANT effects. Functional connectivity foci were labeled by comparison with the Anatomical Automated Labeling atlas in SPM8 (Tzourio-Mazoyer et al., 2002). Voxel coordinates are reported in MNI space.

\section{Results}

\section{Demographics}

Participant demographics are reported in Table 1. The control group reported higher education levels than the PTSD group. Therefore, level of education (ordinally coded) was used a covariate in the analyses below.

\section{ANT results}

Conflict monitoring We found no significant group difference in the magnitude of the conflict effect, $p=.16$ (Table 3 , Fig. 2).

Alerting attention No significant group difference was apparent in the magnitude of the alerting effect, either, $p=.36$ (Table 3, Fig. 2).

Orienting attention The PTSD group showed a larger orienting effect than did controls [Diagnosis $\times$ Cue Type interaction: $F(1,72)=6.31, p=.014$; see Table 3, Fig. 2]. Tests of simple effects revealed a significant difference in RT between the groups in center cue trials $(76.32 \mathrm{~ms}), F(1,72)=$ $5.644, p=.020$, and a trend level difference in spatial cue trials $(60.72 \mathrm{~ms}), F(1,72)=3.624, p=.061$. To control for potential effects of group differences in mean RT on the whole task, we expressed orienting as a percentage of mean RT. The effects remained significant with larger orienting effects in PTSD, $F(1,72)=4.899, p=.030$, driven by slower RT in the center, $F(1,72)=7.462, p=.008$, but not in the spatial $(p=.365)$ cue trials, Additionally, a significant group difference in the orienting effect remained even after removal of the 18 PTSD patients with mTBI, $F(1,54)=7.605, p=.008$.

Accuracy We found no significant group differences in accuracy, $p>.20$ (see Table 4).

\section{MRI results}

Three participants were excluded from the final fMRI analyses due to technical problems in data acquisition (one control and one PTSD participant) and excessive head movement (one control). No group differences in head movement emerged, $p>.1$ for all parameters. Since we found significant group differences only in orienting attention, the subsequent ANT-ICN analyses involved only the association between the orienting effect and (1) DAN connectivity and (2) SN connectivity.

DAN connectivity and the ANT orienting effect Because the DAN is thought to play a key role in orienting attention (Corbetta \& Shulman, 2002), we examined the interaction 
Table 2 Regions of interest for connectivity analyses

\begin{tabular}{lll}
\hline ICN & Seed $(x y z$ Coordinates $)$ & Search Mask $(x y z$ Coordinates $)$ \\
\hline SN & dACC $(-4640)$ & dACC $( \pm 4640)$ \\
& & Insula/Precentral gyrus $( \pm 51-78)$ \\
& Bilateral anatomical amygdala \\
VAN & IFG $(5226-4)$ & IFG $( \pm 5226-4)$ \\
& & Middle temporal gyrus $( \pm 62-37-3)$ \\
DAN & MFG $(46634)$ & MFG $( \pm 46634)$ \\
& & Inferior parietal lobule $(44-4846)$ \\
DMN & N/A & Superior parietal lobule $(-38-5648)$ \\
& & PCC $( \pm 2-5127)$ \\
& & vmPFC $( \pm 254-3)$ \\
\hline
\end{tabular}

Abbreviations: dACC, dorsal anterior cingulate cortex; DAN, dorsal attention network; DMN, default mode network; ICN, intrinsic connectivity network; IFG, inferior frontal gyrus; MFG, middle frontal gyrus; PCC, posterior cingulate cortex; SN, salience network; VAN, ventral attention network; vmPFC, ventromedial prefrontal cortex. 10-mm spheres were created around each coordinate on the basis of (De Luca et al., 2006). The anatomical amygdala $(k=$ 264) was defined by the WFU Pick Atlas (Maldjian, Laurienti, \& Burdette, 2004; Maldjian, Laurienti, Kraft, \& Burdette, 2003; Tzourio-Mazoyer et al., 2002) and included because it has been described as part of the SN (Seeley et al., 2007), and was implicated in previous PTSD rsFC studies (Rabinak et al., 2011; Sripada et al., 2012a; b), but did not appear in the previous rsFC study from which the other SN ROIs were chosen (De Luca et al., 2006).

between the orienting effect, group status, and DAN connectivity, employing a seed in the middle frontal gyrus (MFG; De Luca et al., 2006). We found a significant Group $\times$ Orienting Effect $\times$ MFG-Amygdala interaction . In controls, the orienting effect increased with crossnetwork functional connectivity between the MFG and the SN (right amygdala). In the PTSD group, however, there was no such correlation (Table 5, Fig. 3). On the other hand, the PTSD group displayed greater resting-state functional connectivity between the MFG and the right amygdala without ANT regressors (see the Supplementary materials).

We still observed a peak in the right amygdala after excluding individuals with mTBI in the PTSD group from the Group $\times$ Orienting $\times$ MFG interaction analysis, $p=.068$; this peak no longer reached conventional levels of significance, likely because this analysis excluded more than a third of the PTSD group.
SN connectivity and the ANT orienting effect Relative to the control group, the PTSD group exhibited greater restingstate functional connectivity between the SN (dACC) and the default mode network (vmPFC) independent of attentional performance (Supplementary materials), replicating our previous findings (Sripada et al. 2012a). The control group did not exhibit greater rsFC than the PTSD group between the SN and any other regions.

We also examined whether SN connectivity (dACC seed) correlated with the magnitude of the orienting effect. Across all participants, the orienting effect increased with crossnetwork functional connectivity between the dACC and the VAN (left middle/superior temporal gyrus). However, separate group analyses showed that this was driven entirely by the control group (Table 6). Indeed, we observed multiple significant Group $\times$ Orienting Effect $\times$ dACC Connectivity interactions, driven by the control group exhibiting stronger

Table 3 Reaction times on the ANT in the PTSD and control groups

\begin{tabular}{|c|c|c|c|c|c|}
\hline ANT Component & PTSD $M(\mathrm{~ms}), S D$ & Control $M(\mathrm{~ms}), S D$ & Main Effect of Trial Type $F, p$ & Main Effect of Group $F, p$ & Interaction $F, p$ \\
\hline Alerting & & & $8.32, .005$ & $5.10, .023$ & $0.85, .36$ \\
\hline No cue & $671.38,118.63$ & $599.09,98.57$ & & & \\
\hline Double cue & $629.67,117.31$ & $564.31,93.28$ & & & \\
\hline Orienting & & & $20.38,<.001$ & $4.66, .034$ & $6.31, .014$ \\
\hline Center cue & $650.64,128.27$ & $574.32,105.24$ & & & \\
\hline Spatial cue & $598.13,126.50$ & $537.41,92.57$ & & & \\
\hline Conflict & & & $29.88,<.001$ & $5.18, .026$ & $2.02, .16$ \\
\hline Congruent & $598.88,123.04$ & $536.02,89.19$ & & & \\
\hline Incongruent & $723.68,135.18$ & $643.10,116.19$ & & & \\
\hline
\end{tabular}

Abbreviations: ANT, Attention Network Task. 


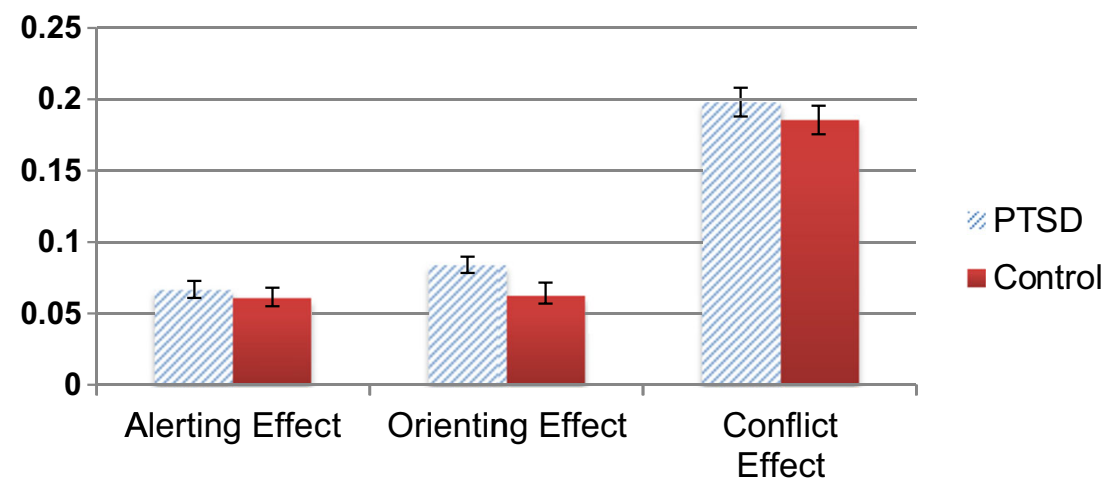

Fig. 2 Group differences in reaction times on the ANT. The PTSD group exhibited a larger orienting effect than did controls ( $p=.014$ for raw RTs, $p=.030$ when controlling for mean RT), but no difference in the magnitudes of the alerting or conflict effects. The results plotted above

relationships between the orienting effect and cross-network connectivity than the PTSD group. More specifically, in the control group, the orienting effect increased with crossnetwork functional connectivity between the dACC seed and (1) the DAN and (2) the DMN. In contrast, in the PTSD group the orienting effect decreased with cross-network connectivity between the dACC seed and the DMN (vmPFC; Fig. 4, Table 6). The peaks from these interactions remained in the $\operatorname{vmPFC}(p=.002 \mathrm{FWE})$, left superior frontal gyrus $(p=.281$ FWE), right superior frontal gyrus ( $p=.459$ FWE), left superior temporal gyrus $(p=.079 \mathrm{FWE})$, and right superior temporal gyrus ( $p=.213 \mathrm{FWE}$ ), after excluding individuals with mTBI in the PTSD group; however, the significance of these effects was reduced, likely because this analysis excluded more than a third of the PTSD group.

To better understand the negative relationship between resting-state functional connectivity and the orienting effect in the PTSD group, we examined variability in these measures using PTSD symptom severity on the CAPS and mTBI status. We found a positive correlation between $\mathrm{dACC}-\mathrm{vmPFC}$ resting-state functional connectivity and intrusive symptoms within the PTSD group, $r(34)=.337, p=.05$ (no such correlation involved the other CAPS subscales, $p>.6$ in all cases). The orienting effect was also negatively correlated with the

Table 4 Accuracy on the ANT in the PTSD and control groups

\begin{tabular}{lllll}
\hline & PTSD $M(\%), S D$ & Control $M(\%), S D$ & $F$ & $p$ \\
\hline All trial types & $96.53,6.57$ & $98.18,1.88$ & 0.926 & .34 \\
No cue & $96.49,6.24$ & $98.24,1.98$ & 1.114 & .30 \\
Double cue & $96.15,6.89$ & $98.18,1.96$ & 1.267 & .26 \\
Center cue & $96.46,7.13$ & $97.92,2.84$ & 0.641 & .43 \\
Spatial cue & $97.14,6.49$ & $98.45,2.09$ & 0.627 & .43 \\
Incongruent & $95.20,10.40$ & $97.14,3.01$ & 0.628 & .43 \\
Congruent & $99.14,1.81$ & $99.60,0.56$ & 0.634 & .43 \\
\hline
\end{tabular}

Abbreviations: ANT, Attention Network Task; PTSD, posttraumatic stress disorder. display the means for each ANT component, normalized by dividing the effect by the mean RT on the task as a whole, plus/minus the standard error of the mean.

CAPS intrusive symptoms subscore (controlling for mean RT, $r=-.380, p=.008$ ). This correlation was driven by the fact that those with greater levels of intrusive symptoms were slower to respond in spatial cue trials $(r=.396, p=.005)$, but not in center cue trials $(r=-.103$, n.s.). Participants in the PTSD group with mTBI, who had higher CAPS scores than those without mTBI, also displayed slower RT in spatial cue trials (Supplemental materials).

\section{Discussion}

The present study yielded two findings that inform our understanding of PTSD. First, relative to age-and-gender-matched controls, PTSD participants exhibited greater orienting effects (which were driven by slower responses to central cue trials). Second, in control but not PTSD participants, cross-network connectivity was associated with greater ANT orienting effects. These findings suggest that the processes underlying the ability to orient spatial attention might be altered in PTSD. To our knowledge, this is the first study to examine relationships between neurocognitive measures of attentional performance and rsFC in PTSD.

\section{Attentional performance and PTSD}

The larger orienting effect we observed in PTSD could reflect (1) a reduced ability to disengage from the center cue location and/or (2) an increased ability to utilize spatial cues (Fig. 5; Fan \& Posner, 2004). In our data, the larger orienting effect in the PTSD group was driven by slower RT in center cue trials, indicating difficulty with disengaging attention from the central cue location and reorienting to the target location (Fan \& Posner, 2004). Because the standard version of the ANT we used does not include invalid cues, we were unable to directly assess disengagement. Nonetheless, our findings are consistent with reports of disengagement impairments using the 
Table 5 Correlations of DAN (MFG seed) connectivity with the orienting effect

\begin{tabular}{|c|c|c|c|c|}
\hline Contrast Map and Brain Region & Cluster Size & MNI Coordinates $(x y z)$ & Analysis $(z)$ & $p(\mathrm{SVC})$ \\
\hline All Participants & & No significant clusters & & \\
\hline PTSD & & No significant clusters & & \\
\hline \multicolumn{5}{|l|}{ Control } \\
\hline Right amygdala & 9 & $18-1-14$ & 3.40 & .032 \\
\hline PTSD > Control & & No significant clusters & & \\
\hline \multicolumn{5}{|l|}{ Control > PTSD } \\
\hline Right amygdala & 14 & $18-1-14$ & 3.38 & .047 \\
\hline
\end{tabular}

Abbreviations: MNI, Montreal Neurological Institute; PTSD, posttraumatic stress disorder; SVC, small volume correction. All correlations are positive.

ANT in a cohort with mixed anxiety disorders (generalized, social, specific phobia, obsessive-compulsive disorder, agoraphobia, and PTSD; Pacheco-Unguetti, Acosta, Marqués, \& Lupiáñez, 2011), and in male veterans with PTSD and mTBI (Barlow-Ogden \& Poynter, 2012). Problems with disengaging and shifting attention away from salient stimuli like threat or trauma cues may be a mechanism underlying PTSD symptom development (Aupperle et al., 2012). Our findings suggest that such problems are not specific to threatening stimuli. Rather, they may reflect a more general impairment of basic attentional processes that operate even on neutral stimuli. Alternatively, the deficits we have observed might comprise a predisposing risk factor for developing PTSD. Future longitudinal studies could be useful in making this distinction.

On the other hand, we found no evidence of increased utilization on spatial cue trials in PTSD. In fact, the PTSD group was nominally slower than controls to respond in spatial cue trials. These findings contrast with a previous report of increased utilization of spatial cues following an anxiety induction in healthy individuals (Garner, Attwood, Baldwin, \& Munafò, 2012), and theoretical reports of facilitated orienting as a mechanism underlying attention biases (Weierich et al.,
2008). Our findings suggest that these effects might be limited to healthy individuals or relevant to threat stimuli only. Our results, however, are consistent with the neuropsychology literature describing PTSD deficits on the trail-making test, part B (Gurvits, 2000; Hart et al., 2008; Jenkins et al., 2000; Koenen et al., 2001; Koso \& Hansen, 2006; LaGarde et al., 2010; Polak et al., 2012; Stein, Kennedy, \& Twamley, 2002; Sutker, Vasterling, Brailey, \& Allain, 1995) and the digitsymbol test (Brandes et al., 2002; Hart et al., 2008; Jenkins et al., 2000; Parslow \& Jorm, 2007). Successful completion of these tasks requires the participant to visually scan information and move his/her attention to a new spatial location.

We were unable to replicate the findings of Leskin and White (2007), who reported that college students with PTSD had deficits with conflict monitoring, but not with orienting attention. Between-study differences (e.g., age, gender, population, and symptom severity) could potentially account for the observed differences. Moreover, the smaller group size in their study could have reduced statistical power for detecting group differences in orienting attention. Clearly, additional research will be needed to clarify the influence of such factors on the nature of attentional deficits in PTSD.

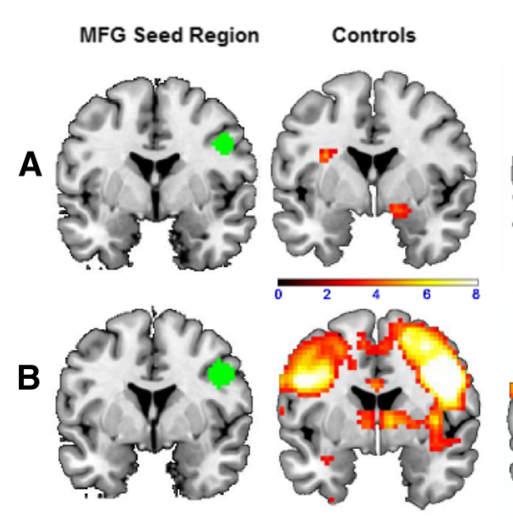

Fig. 3 Functional connectivity analysis of the DAN. (A) Correlations of DAN connectivity with the orienting effect. The orienting effect increased with cross-network functional connectivity between the DAN (right MFG seed) and the SN (right amygdala) in the control group, but we found no such relationship in the PTSD group. (B) Functional connectivity of the

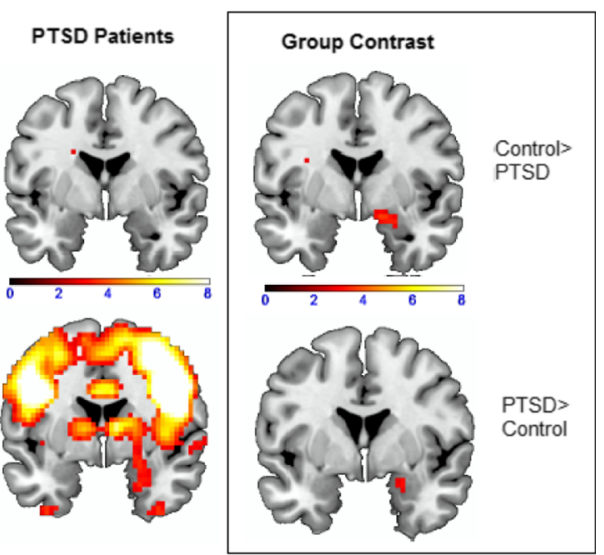

MFG seed without ANT regressors. PTSD patients showed greater connectivity between the MFG seed and the right amygdala than did the controls. Slices are displayed at the Montreal Neurological Institute coordinates $y=-1$ (top row), and $y=1$ (bottom row). The results are corrected for multiple comparisons within regions of interest. 
Table 6 Correlations of SN (dACC seed) connectivity with the orienting effect

\begin{tabular}{|c|c|c|c|c|}
\hline Contrast Map and Brain Region & Cluster Size & MNI Coordinates $(x y z)$ & Analysis $(z)$ & $p$ Value (FWE, FDR) \\
\hline \multicolumn{5}{|l|}{ All Participants } \\
\hline VAN & & & & $.317, .324$ \\
\hline L mid/sup temporal gyrus & 46 & $-60-341$ & 3.06 & (.041 SVC with VAN mask) \\
\hline \multicolumn{5}{|l|}{ PTSD } \\
\hline \multicolumn{5}{|l|}{$\mathrm{DMN}$} \\
\hline vmPFC* & 28 & $2150-2$ & 3.30 & $\begin{array}{l}.620, .599 \\
(.025 \text { SVC with DMN mask) }\end{array}$ \\
\hline \multicolumn{5}{|l|}{ Control } \\
\hline \multicolumn{5}{|l|}{$\mathrm{DMN}$} \\
\hline vmPFC & 362 & $938-11$ & 3.93 & $<.001,<.001$ \\
\hline Precuneus & 361 & $-15-464$ & 3.70 & $<.001,<.001$ \\
\hline L parahipp/hipp & 124 & $-27-19-23$ & 4.21 & $.118, .035$ \\
\hline R parahipp/hipp & 203 & $33-7-38$ & 4.09 & $.014, .005$ \\
\hline \multicolumn{5}{|l|}{ DMN/DAN } \\
\hline $\mathrm{L}$ angular gyrus & 359 & $-27-7958$ & 4.10 & $<.001,<.001$ \\
\hline $\mathrm{R}$ angular gyrus & 106 & $51-6434$ & 3.59 & $.199, .048$ \\
\hline \multicolumn{5}{|l|}{ VAN } \\
\hline L sup temporal gyrus & 112 & $-2711-47$ & 4.34 & $.167, .045$ \\
\hline $\mathrm{R}$ mid/inf/sup temporal gyrus & 528 & $60-10-29$ & 4.45 & $<.001,<.001$ \\
\hline $\mathrm{R}$ inf frontal gyrus & 136 & $4220-35$ & 3.65 & $.084, .048$ \\
\hline PTSD > Controls & & No significant clusters & & \\
\hline \multicolumn{5}{|l|}{ Controls $>$ PTSD } \\
\hline \multicolumn{5}{|l|}{$\mathrm{DMN}$} \\
\hline vmPFC & 342 & 12471 & 4.17 & $.002, .005$ \\
\hline PCC & 118 & $-12-3428$ & 3.62 & $.242, .086$ \\
\hline \multicolumn{5}{|l|}{ DAN } \\
\hline L sup frontal gyrus & 216 & -33270 & 3.81 & $.027, .014$ \\
\hline $\mathrm{R}$ sup frontal gyrus & 232 & 242955 & 3.97 & $.019, .014$ \\
\hline \multicolumn{5}{|l|}{ VAN } \\
\hline L sup temporal gyrus & 182 & $-3320-38$ & 3.72 & $.057, .025$ \\
\hline $\mathrm{R}$ mid/inf temporal gyrus & 241 & $60-16-41$ & 3.81 & $.027, .015$ \\
\hline
\end{tabular}

Abbreviations: DAN, dorsal attention network; FDR, false discovery rate; FWE, family-wise error; Hipp, hippocampus; Inf, inferior; L, left; Mid, middle; MNI, Montreal Neurological Institute; Parahipp, parahippocampal gyrus; PCC, posterior cingulate cortex; R, right; SN, salience network; Sup; superior; VAN, ventral attention network; vmPFC, ventromedial prefrontal cortex. *Negative correlation in PTSD group between orienting effect and dACC-vmPFC connectivity. All other correlations in the table are positive.

\section{Attentional performance and intrinsic network connectivity}

We observed a positive relationship between the orienting effect and cross-network connectivity in the control group. First, there was a positive relationship between the orienting effect and SN cross-network connectivity (dACC-default mode, dACC-dorsal attention, dACC-ventral attention). Second, we found a positive relationship between the orienting effect and DAN cross-network connectivity (MFG-SN). Interestingly, larger orienting effects in the control group were driven by slower RT in center cue trials
(Supplementary materials, same pattern as PTSD vs. control group finding), suggesting that the cross-network connectivity effects above might reflect difficulty with disengaging attention from the center of the display. Consistent with this possibility, the SN and attention networks might be involved in DMN disengagement when salient stimuli are present (Daniels et al., 2010).

Another possibility is that cross-network connectivity leads to difficulties with reorienting, whereby SN intrusions upon other ICNs interfere with the DAN's and VAN's abilities to focus attention on the task at hand. The temporoparietal junction has been implicated in reorienting (Corbetta \& Shulman, 


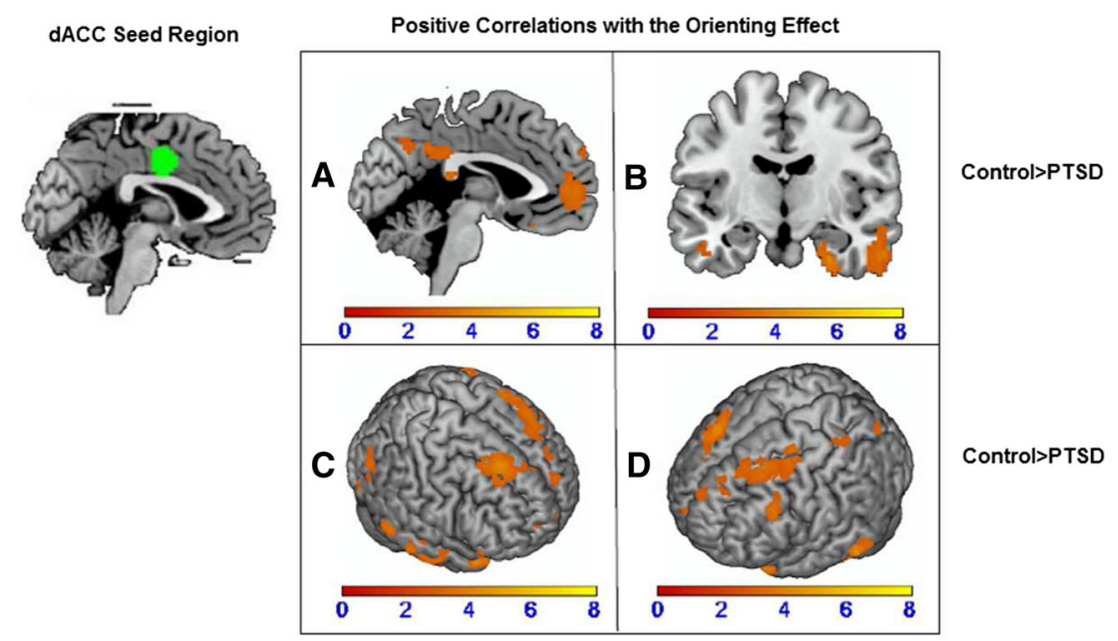

Fig. 4 Interaction of SN Connectivity $\times$ Orienting Effect $\times$ Group Status. Controls showed greater connectivity correlated with the orienting effect than did PTSD patients between the AACC seed and several regions of the $\mathrm{DMN}$, including (A) the PCC and vmPFC and (B) the right hippocampus, as well as the DAN (C, D) in the bilateral superior frontal gyrus, and several regions of the VAN $(\mathrm{B}, \mathrm{C}, \mathrm{D})$, including the middle and superior temporal gyri. Images A and B are displayed at Montreal Neurological Institute coordinates $x=-3$ and $y=-10$, respectfully, and images $\mathrm{C}$ and $\mathrm{D}$ show whole-brain connectivity, displayed at azimuth $120^{\circ}$, elevation $45^{\circ}$, and azimuth $240^{\circ}$, elevation $45^{\circ}$, respectively. The results are corrected for multiple comparisons within regions of interest using FWE $p<.05$ at the cluster level.
2002), consistent with our findings that orienting effects were associated with greater dACC-left middle/superior temporal gyrus connectivity. Future studies will need to investigate whether SN cross-network connectivity on-task is related to difficulty with disengaging or reorienting attention.

Interestingly, we did not detect the same relationships between the orienting effect and cross-network connectivity in PTSD patients. First, we found no relationship between the orienting effect and dACC-DAN, dACC-VAN, or MFG-SN connectivity. Because this pattern was observed in the control group, normative relationships between ICNs appear to be disrupted in PTSD. It is possible that attentional disengagement in PTSD is related to the connectivity of other neural structures in which we did not place seeds, such as the frontal eye fields and TPJ that are involved in shifting attention (Corbetta \& Shulman, 2002). Future researchers might therefore employ a connectomics approach, rather than the present seed-based approach, to investigate ICNs.

We also found a negative relationship between the orienting effect and $\mathrm{dACC}-\mathrm{vmPFC}$ connectivity, which was different from what we observed in the control group. In the control group, disengagement of attention was positively correlated with $\mathrm{dACC}-\mathrm{vmPFC}$ connectivity. One possible explanation for this seemingly discrepant finding, is that orienting to spatial cues, as well as disengagement from center cues, contributes to dACC-VmPFC connectivity in PTSD patients. When examining variability within the PTSD group, we found that greater intrusive symptoms were associated with slower $\mathrm{RT}$ on spatial cue trials and greater dACC-vmPFC connectivity.

Slower RT on spatial cue trials is reflective of a difficulty reorienting to the target location. This is an additional finding above and beyond the between group difference in disengagement that we observed. It is possible that difficulties with disengagement are a precursor to PTSD, or develop early on in the course of PTSD. Other factors such as mTBI or PTSD severity may then contribute to an additional deficit in the reorienting of spatial attention. Supporting this, we found that PTSD participants with mTBI exhibited slower RT to spatial cue trials compared those without mTBI. This is consistent
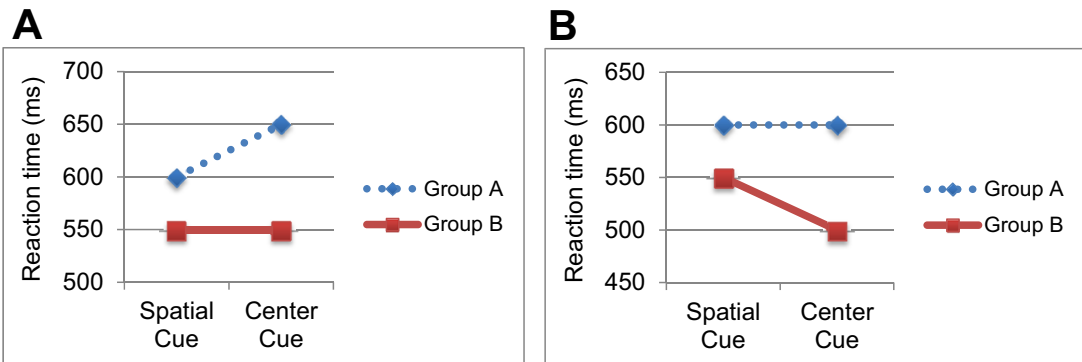

Fig. 5 Hypothetical data showing how a group difference in the orienting effect could be driven by (A) disengagement or (B) spatial orienting. In our data, the PTSD group had a larger orienting effect than did the control group, driven by slower RTs to center-cue trials, consistent with panel A. 


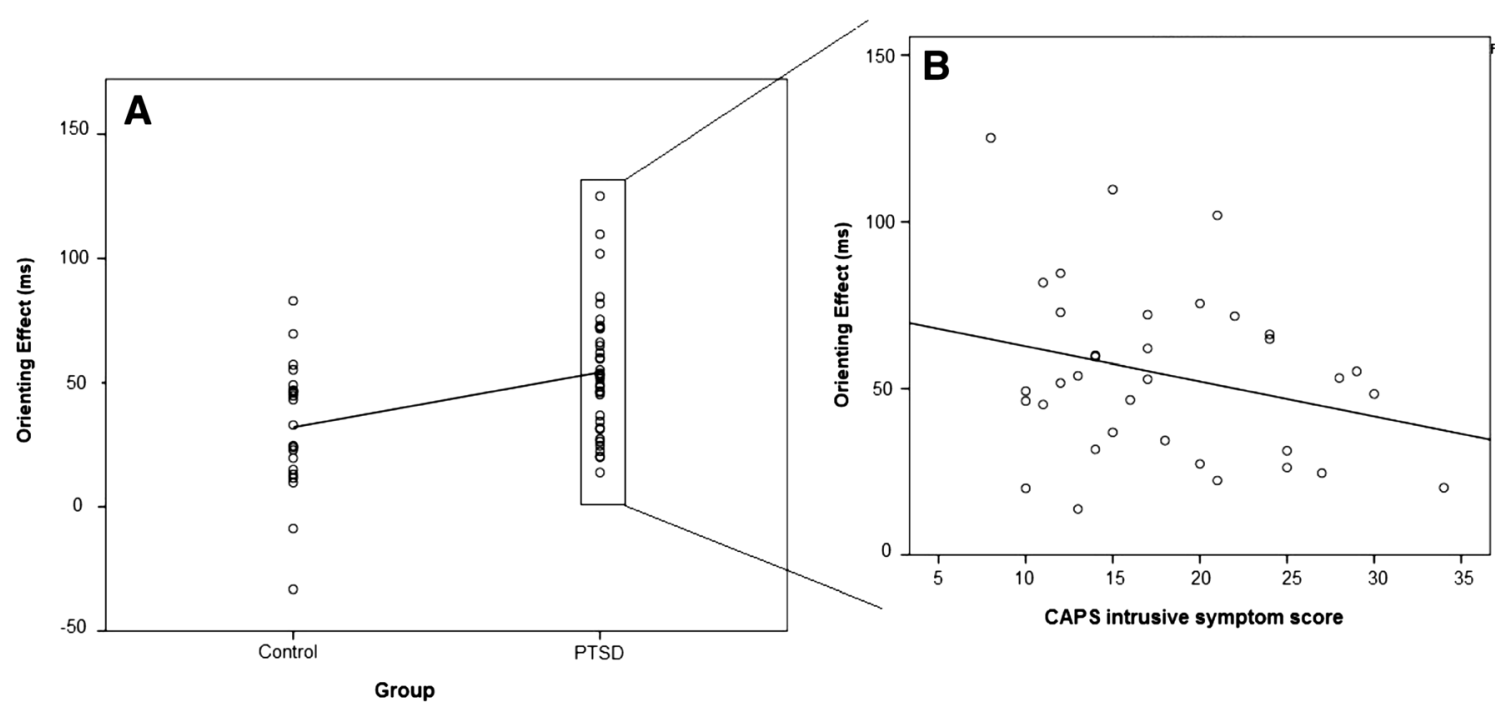

Fig. 6 Magnitude of the orienting effect between and within groups. (A) The PTSD group displayed a larger average orienting effect than did the control group. (B) Within the PTSD group, however, greater intrusive symptoms were associated with smaller orienting effects.

with reports of orienting attention deficits following concussion (Pavlovskaya, Groswasser, Keren, Mordvinov, \& Hochstein, 2007; van Donkelaar et al., 2005).

Greater connectivity between the dACC-vmPFC in the PTSD group could also reflect intrusive thoughts interfering with the ability of the SN to focus on spatial cues. The vmPFC is part of the DMN, that is thought to support stimulusindependent thought, such as day-dreaming and selfreferential processing (Buckner \& Vincent, 2007), and thus might be associated with intrusive thoughts as well. This could contribute to slower RT on spatial cue trials and the apparent "contradiction" whereby the orienting effect was larger in the PTSD group than controls, but negatively correlated with intrusive symptoms (Fig. 6). The seeming contradiction is simply the outcome of how the orienting effect is calculated - the difference in RT between the spatial and the central cue trials. In both groups, increased connectivity between the SN and DMN may contribute to slower RTs - in controls by slowing mainly responses to central cues, and in PTSD patients for whom disengagement is already affected, it may also slow responses to spatial cues.

We also replicated earlier findings (Brown et al., 2014; Lanius et al., 2010; Sripada et al. 2012b; Zhang et al., 2015) of greater salience to default mode network (dACC-vmPFC) connectivity in PTSD. This was independent of ANT performance. We further extended this to show greater SN-DAN (right amygdala to right MFG) connectivity in PTSD independent of ANT performance (Supplementary materials). It is possible that SN intrusions upon the DAN may also contribute to difficulty orienting. Our findings were specific to the right amygdala, consistent with Barlow-Ogden \& Poynter (2012) who found that orienting deficits in veterans with mTBI and PTSD were specific to the right hemisphere.
Together our findings support a notion that the normal relationship between ICNs and orienting attention is disrupted in PTSD. Recent evidence points to ICN segregation as being important for normal cognitive development and functioning (Kelly, Uddin, Biswal, Castellanos, \& Milham, 2008; Mennes et al., 2010), suggesting that abnormal connectivity patterns might contribute to PTSD pathophysiology.

\section{Limitations}

Several limitations of the present study merit consideration. First, because we used community, rather than combatexposed controls, it is possible that our findings are related to differences between military and nonmilitary personnel. If our findings were due to military training, we would expect the PTSD group to show better performance than the controls, which we did not observe, because heightened attention is heavily emphasized in military training (Messinger, 2013). Because IQ and general cognitive ability have been linked to attention performance (Schweizer \& Moosebrugger, 2004) and cross-network connectivity (van den Heuvel, Stam, Kahn, \& Hulshoff Pol, 2009), it is also possible our the findings are due to the differences in educational attainment between the two groups; however the findings held after controlling for level of education, which is correlated with IQ (Winship \& Korenman, 1997). The findings could also be due to group differences in trauma exposure. Consistent with epidemiological studies (Kessler, Sonnega, Bromet, Hughes, \& Nelson, 1995), 50\% of our controls had experienced traumatic events, and no significant differences in ANT performance emerged between the subgroups (Supplementary material), but future work with combat-exposed controls will be able to clarify whether our findings are partially related to 
trauma exposure. Second, the sample sizes of the control and PTSD groups were unequal; however, this was accounted for by statistical analyses that do not assume equal variance. Third, concerns have been raised regarding the psychometric properties of the ANT (MacLeod et al., 2010). Past studies have found small interactions between the networks, however, we found them to be independent of one another $(p>.1)$. Furthermore, in rsFC studies, it is difficult to determine the exact cognitive processes involved in the "rest" scan, which could contribute to group differences in rsFC. Lastly, all our participants were male and our sample size was moderate. Future studies will be needed to determine whether our findings extend to females.

\section{Conclusion}

Our results demonstrate that greater cross-network connectivity involving the $\mathrm{SN}$ is associated with impairments in the disengagement and orienting of attention, processes that were altered in PTSD. These findings might suggest that both attentional orienting processes and the balance that is normally observed between the ICNs might be disrupted in veterans with PTSD. Interventions that utilize attention training, such as mindfulness (Shapiro, Carlson, Astin, \& Freedman, 2006) might be useful for alleviating attentional impairments in PTSD. Further, our results suggest that interventions targeting orienting and disengagement deficits specifically may be a new avenue for PTSD research.

\begin{abstract}
Author note Data previously presented as a poster at the Anxiety and Depression Association of America and the Society of Biological Psychiatry Annual Conferences, 2015. We thank Mike Angstadt, Daniel Kessler, Chandra Sripada and The University of Michigan Neuroimaging Methods Core for technical support. We thank Stephen Taylor and Elizabeth Duval for personal support. We thank Birgit Roller, Nita Patel, Meagan Lewis and Yanli Lin for assistance with data collection. This project was supported in part by a Department of Defense Grant W81XWH082020a8 to I.L. and A.P.K., and the Mind and Life Institute Varela Award to A.P.K. S.R.B. is supported by the University of Michigan Psychology Department and has no potential conflict of interest. A.P.K. has been supported by the Department of Defense and the Mind and Life Institute and declares no conflict of interest. R.K.S. is supported by the University of Michigan Psychiatry Department and the Ann Arbor VA, and declares no conflict of interest. D.H.W. has no financial relationships to disclose. R.W. has no financial relationships to disclose. I.L. has been supported by the Department of Defense and declares no conflict of interest.
\end{abstract}

\section{References}

Anderson, J. S., Druzgal, T. J., Lopez-Larson, M., Jeong, E.-K., Desai, K., \& Yurgelun-Todd, D. (2011). Network anticorrelations, global regression, and phase-shifted soft tissue correction. Human Brain Mapping, 32, 919-934. doi:10.1002/hbm.21079
Aupperle, R. L., Melrose, A. J., Stein, M. B., \& Paulus, M. P. (2012). Executive function and PTSD: Disengaging from trauma. Neuropharmacology, 62, 686-694. doi:10.1016/j. neuropharm.2011.02.008

Barlow-Ogden, K., Poynter, W. (2012). Mild traumatic brain injury and posttraumatic stress disorder: Investigation of visual attention in Operation Iraqi Freedom/Operation Enduring Freedom veterans. The Journal of Rehabilitation Research and Development, 49(7), 1101

Blake, D. D., Weathers, F. W., Nagy, L. M., Kaloupek, D. G., Gusman, F. D., Charney, D. S., \& Keane, T. M. (1995). The development of a Clinician-Administered PTSD Scale. Journal of Traumatic Stress, 8 , 75-90.

Botvinick, M. M., Cohen, J. D., \& Carter, C. S. (2004). Conflict monitoring and anterior cingulate cortex: An update. Trends in Cognitive Sciences, 8, 539-546. doi:10.1016/j.tics.2004.10.003

Brandes, D., Ben-Schachar, G., Gilboa, A., Bonne, O., Freedman, S., \& Shalev, A. Y. (2002). PTSD symptoms and cognitive performance in recent trauma survivors. Psychiatry Research, 110, 231-238. doi:10.1016/S0165-1781(02)00125-7

Brock, S. E., \& Clinton, A. (2007). Diagnosis of attention-deficit/hyperactivity disorder $(\mathrm{AD} / \mathrm{HD})$ in childhood: A review of the literature. California School Psychologist, 12, 73-91. doi:10.1007 /BF03340933

Brown, V. M., Labar, K. S., Haswell, C. C., Gold, A. L., Mid-Atlantic MIRECC Workgroup, Beall, S. K., . . . Morey, R. A. (2014). Altered resting-state functional connectivity of basolateral and centromedial amygdala complexes in posttraumatic stress disorder. Neuropsychopharmacology, 39, 361-369. doi:10.1038 /npp.2013.197

Buckner, R. L., \& Vincent, J. L. (2007). Unrest at rest: Default activity and spontaneous network correlations. NeuroImage, 37, 1091-6-9. doi:10.1016/j.neuroimage.2007.01.010

Cao, Q., Zang, Y., Zhu, C., Cao, X., Sun, L., Zhou, X., \& Wang, Y. (2008). Alerting deficits in children with attention deficit/ hyperactivity disorder: Event-related fMRI evidence. Brain Research, 1219, 159-168. doi:10.1016/j.brainres.2008.04.028

Corbetta, M., \& Shulman, G. L. (2002). Control of goal-directed and stimulus-driven attention in the brain. Nature Reviews Neuroscience, 3, 201-215. doi:10.1038/nrn755

Daniels, J. K., McFarlane, A. C., Bluhm, R. L., Moores, K. A., Clark, C. R., Shaw, M. E., . . . Lanius, R. A. (2010). Switching between executive and default mode networks in posttraumatic stress disorder: Alterations in functional connectivity. Journal of Psychiatry and Neuroscience, 35, 258-266.

De Bellis, M. D., Woolley, D. P., \& Hooper, S. R. (2013). Neuropsychological findings in pediatric maltreatment: Relationship of PTSD, dissociative symptoms, and abuse/neglect indices to neurocognitive outcomes. Child Maltreatment, 18, 171183. doi: $10.1177 / 1077559513497420$

De Luca, M., Beckmann, C. F., De Stefano, N., Matthews, P. M., \& Smith, S. M. (2006). fMRI resting state networks define distinct modes of long-distance interactions in the human brain. NeuroImage, 29, 1359-1367. doi:10.1016/j. neuroimage.2005.08.035

Eren-Koçak, E., Kılıç, C., Aydın, I., \& Hızlı, F. G. (2009). Memory and prefrontal functions in earthquake survivors: Differences between current and past post-traumatic stress disorder patients. Acta Psychiatrica Scandinavica, 119, 35-44. doi:10.1111/j.16000447.2008.01281.x

Eriksen, B. A., \& Eriksen, C. W. (1974). Effects of noise letters upon the identification of a target letter in a nonsearch task. Perception \& Psychophysics, 16, 143-149. doi:10.3758/BF03203267

Fair, D. A., Nigg, J. T., Iyer, S., Bathula, D., Mills, K. L., Dosenbach, N. U. F., . . Milham, M. P. (2012). Distinct neural signatures detected for ADHD subtypes after controlling for micro-movements in 
resting state functional connectivity MRI data. Frontiers in Systems Neuroscience, 6, 80. doi:10.3389/fnsys.2012.00080

Fan, J., McCandliss, B. D., Fossella, J., Flombaum, J. I., \& Posner, M. I. (2005). The activation of attentional networks. Neurolmage, 26, 471-479. doi:10.1016/j.neuroimage.2005.02.004

Fan, J., McCandliss, B. D., Sommer, T., Raz, A., \& Posner, M. I. (2002). Testing the efficiency and independence of attentional networks. Journal of Cognitive Neuroscience, 14, 340-347.

Fan, J., \& Posner, M. (2004). Human attentional networks. Psychiatrische Praxis, 31(Suppl. 2), 5210-5214. Retrieved from http://people.qc.cuny.edu/Faculty/Jin. Fan/Documents/Fan2004_31.pdf

Fischer, E. H., Dornelas, E. A., \& Goethe, J. W. (2001). Characteristics of people lost to attrition in psychiatric follow-up studies. Journal of Nervous and Mental Disease, 189, 49-55.

Fox, M. D., \& Greicius, M. (2010). Clinical applications of resting state functional connectivity. Frontiers in Systems Neuroscience, 4, 19. doi:10.3389/fnsys.2010.00019

Fox, M. D., Snyder, A. Z., Vincent, J. L., Corbetta, M., Van Essen, D. C., \& Raichle, M. E. (2005). The human brain is intrinsically organized into dynamic, anticorrelated functional networks. Proceedings of the National Academy of Sciences, 102, 9673-9678.

Garner, M., Attwood, A., Baldwin, D. S., \& Munafò, M. R. (2012). Inhalation of $7.5 \%$ carbon dioxide increases alerting and orienting attention network function. Psychopharmacology, 223, 67-73. doi:10.1007/s00213-012-2690-4

Gong, Q., Li, L., Du, M., Pettersson-Yeo, W., Crossley, N., Yang, X., . . . Mechelli, A. (2014). Quantitative prediction of individual psychopathology in trauma survivors using resting-state FMRI. Neuropsychopharmacology, 39, 681-687. doi:10.1038 /npp.2013.251

Gurvits, T. (2000). Neurologic soft signs in chronic posttraumatic stress disorder. Archives of General Psychiatry, 57, 181-186. doi:10.1001 /archpsyc.57.2.181

Hart, J., Kimbrell, T., Fauver, P., Cherry, B. J., Pitcock, J., Booe, L. Q., . . . Freeman, T. W. (2008). Cognitive dysfunctions associated with PTSD: Evidence from World War II prisoners of war. Journal of Neuropsychiatry and Clinical Neurosciences, 20, 309-316. doi:10.1176/jnp.2008.20.3.309

Jenkins, M. A., Langlais, P. J., Delis, D., \& Cohen, R. A. (2000). Attentional dysfunction associated with posttraumatic stress disorder among rape survivors. Clinical Neuropsychologist, 14, 7-12.

Jha, A. P., Krompinger, J., \& Baime, M. J. (2007). Mindfulness training modifies subsystems of attention. Cognitive, Affective, \& Behavioral Neuroscience, 7, 109-119. doi:10.3758/CABN.7.2.109

Johnsen, G. E., \& Asbjørnsen, A. E. (2008). Consistent impaired verbal memory in PTSD: A meta-analysis. Journal of Affective Disorders, 111, 74-82. doi:10.1016/j.jad.2008.02.007

Kelly, A. M. C., Uddin, L. Q., Biswal, B. B., Castellanos, F. X., \& Milham, M. P. (2008). Competition between functional brain networks mediates behavioral variability. NeuroImage, 39, 527-537. doi:10.1016/j.neuroimage.2007.08.008

Kennis, M., Rademaker, A. R., van Rooij, S. J. H., Kahn, R. S., \& Geuze, E. (2015). Resting state functional connectivity of the anterior cingulate cortex in veterans with and without post-traumatic stress disorder. Human Brain Mapping, 36, 99-109. doi:10.1002/hbm.22615

Kennis, M., van Rooij, S. J. H., van den Heuvel, M. P., Kahn, R. S., \& Geuze, E. (2016). Functional network topology associated with posttraumatic stress disorder in veterans. NeuroImage: Clinical, 10, 302-309. doi:10.1016/j.nicl.2015.12.008

Kessler, R. C., Sonnega, A., Bromet, E., Hughes, M., \& Nelson, C. B. (1995). Posttraumatic stress disorder in the National Comorbidity Survey. Archives of General Psychiatry, 52, 1048-1060.

King, A. P., Block, S. R., Sripada, R. K., Rauch, S. A. M., Porter, K. E., Favorite, T. K., . . . Liberzon, I. (2016). A pilot study of mindfulnessbased exposure therapy in OEF/OIF combat veterans with PTSD:
Altered medial frontal cortex and amygdala responses in socialemotional processing. Frontiers in Psychiatry, 7, 154. doi: 10.3389/FPSYT.2016.00154

Koenen, K. C., Driver, K. L., Oscar-Berman, M., Wolfe, J., Folsom, S., Huang, M. T., \& Schlesinger, L. (2001). Measures of prefrontal system dysfunction in posttraumatic stress disorder. Brain and Cognition, 45, 64-78. doi:10.1006/brcg.2000.1256

Koso, M., \& Hansen, S. (2006). Executive function and memory in posttraumatic stress disorder: A study of Bosnian war veterans. European Psychiatry, 21, 167-173. doi:10.1016/j. eurpsy.2005.06.004

Kucyi, A., Hodaie, M., \& Davis, K. D. (2012). Lateralization in intrinsic functional connectivity of the temporoparietal junction with salience- and attention-related brain networks. Journal of Neurophysiology, 108, 3382-3392. doi:10.1152/jn.00674.2012

LaGarde, G., Doyon, J., \& Brunet, A. (2010). Memory and executive dysfunctions associated with acute posttraumatic stress disorder. Psychiatry Research, 177, 144-149. doi:10.1016/j. psychres.2009.02.002

Lanius, R. A., Bluhm, R. L., Coupland, N. J., Hegadoren, K. M., Rowe, B., Théberge, J., . . . Brimson, M. (2010). Default mode network connectivity as a predictor of post-traumatic stress disorder symptom severity in acutely traumatized subjects. Acta Psychiatrica Scandinavica, 121, 33-40. doi:10.1111/j.1600-0447.2009.01391.x

Leskin, L. P., \& White, P. M. (2007). Attentional networks reveal executive function deficits in posttraumatic stress disorder. Neuropsychology, 21, 275-284. doi:10.1037/0894-4105.21.3.275

Lund, T. E., Madsen, K. H., Sidaros, K., Luo, W.-L., \& Nichols, T. E. (2006). Non-white noise in fMRI: Does modelling have an impact? NeuroImage, 29, 54-66. doi:10.1016/j.neuroimage.2005.07.005

MacLeod, J. W., Lawrence, M. A., McConnell, M. M., Eskes, G. A., Klein, R. M., \& Shore, D. I. (2010). Appraising the ANT: Psychometric and theoretical considerations of the Attention Network Test. Neuropsychology, 24, 637-651. doi:10.1037 /a0019803

Maldjian, J. A., Laurienti, P. J., Kraft, R. A., \& Burdette, J. H. (2003). An automated method for neuroanatomic and cytoarchitectonic atlasbased interrogation of fMRI data sets. NeuroImage, 19, 12331239. doi:10.1016/S1053-811900169-1

Maldjian, J. A., Laurienti, P. J., \& Burdette, J. H. (2004). Precentral gyrus discrepancy in electronic versions of the Talairach atlas. NeuroImage, 21, 450-455. doi:10.1016/j.neuroimage.2003.09.032

Mennes, M., Kelly, C., Zuo, X.-N., Di Martino, A., Biswal, B. B., Castellanos, F. X., \& Milham, M. P. (2010). Inter-individual differences in resting-state functional connectivity predict task-induced BOLD activity. NeuroImage, 50, 1690-1701. doi:10.1016/j. neuroimage.2010.01.002

Messinger, S. D. (2013). Vigilance and attention among U.S. service members and veterans after combat. Anthropology of Consciousness, 24, 191-207. doi:10.1111/anoc.12013

Muto, V., Shaffii-Le Bourdiec, A., Matarazzo, L., Foret, A., Mascetti, L., Jaspar, M., ... Maquet, P. (2012). Influence of acute sleep loss on the neural correlates of alerting, orientating and executive attention components. Journal of Sleep Research, 21, 648-658. doi:10.1111 j.1365-2869.2012.01020.x

Nicholson, A. A., Sapru, I., Densmore, M., Frewen, P. A., Neufeld, R. W. J., Théberge, J., . . Lanius, R. A. (2016). Unique insula subregion resting-state functional connectivity with amygdala complexes in posttraumatic stress disorder and its dissociative subtype. Psychiatry Research, 250, 61-72. doi:10.1016/j. pscychresns.2016.02.002

Ogden, K. B. (2010). Traumatic brain injury and posttraumatic stress disorder: A quantitative investigation of vision and attention. Unpublished master's thesis, Western Carolina University, Cullowhee, NC. Retrieved from http://libres.uncg. edu/ir/wcu/f/ogden2010.pdf 
Pacheco-Unguetti, A. P., Acosta, A., Marqués, E., \& Lupiáñez, J. (2011). Alterations of the attentional networks in patients with anxiety disorders. Journal of Anxiety Disorders, 25, 888-895. doi:10.1016/j. janxdis.2011.04.010

Parslow, R. A., \& Jorm, A. F. (2007). Pretrauma and posttrauma neurocognitive functioning and PTSD symptoms in a community sample of young adults. American Journal of Psychiatry, 164, 509515. doi:10.1176/appi.ajp.164.3.509

Pavlovskaya, M., Groswasser, Z., Keren, O., Mordvinov, E., \& Hochstein, S. (2007). Hemispheric visual atentional imbalance in patients with traumatic brain injury. Brain and Cognition, 64, 2129. doi:10.1016/j.bandc.2006.10.003

Perry, R. J., \& Hodges, J. R. (1999). Attention and executive deficits in Alzheimer's disease: A critical review. Brain, 122, 383-404. doi:10.1093/brain/122.3.383

Petersen, S. E., \& Posner, M. I. (2012). The attention system of the human brain: 20 years after. Annual Review of Neuroscience, 35, 73-89. doi:10.1146/annurev-neuro-062111-150525

Pineles, S. L., Shipherd, J. C., Mostoufia, S. M., Abramovitza, S. M., \& Yovelc, I. (2009). Attentional biases in PTSD: More evidence for interference. Behaviour Research and Therapy, 47, 1050-1057. doi:10.1016/j.brat.2009.08.001

Polak, A. R., Witteveen, A. B., Reitsma, J. B., \& Olff, M. (2012). The role of executive function in posttraumatic stress disorder: A systematic review. Journal of Affective Disorders, 141, 11-21. doi:10.1016/j. jad.2012.01.001

Posner, M. I. (1980). Orienting of attention. Quarterly Journal of Experimental Psychology, 32, 3-25. doi:10.1080 /00335558008248231

Posner, M. I., \& Petersen, S. E. (1990). The attention system of the human brain. Annual Review of Neuroscience, 13, 25-42. doi: 10.1146 /annurev.ne.13.030190.000325

Power, J. D., Barnes, K. A., Snyder, A. Z., Schlaggar, B. L., \& Petersen, S. E. (2012). Spurious but systematic correlations in functional connectivity MRI networks arise from subject motion. NeuroImage, 59, 2142-2154. doi:10.1016/j.neuroimage.2011.10.018

Qureshi, S. U., Long, M. E., Bradshaw, M. R., Pyne, J. M., Magruder, K. M., Kimbrell, T., . . . Kunik, M. E. (2011). Does PTSD impair cognition beyond the effect of trauma? Journal of Neuropsychiatry and Clinical Neurosciences, 23, 16-28.

Rabinak, C. A., Angstadt, M., Welsh, R. C., Kenndy, A. E., Lyubkin, M., Martis, B., \& Phan, K. L. (2011). Altered amygdala resting-state functional connectivity in post-traumatic stress disorder. Frontiers in Psychiatry, 2, 62. doi:10.3389/fpsyt.2011.00062

Raichle, M. E. (2011). The restless brain. Brain Connectivity, 1, 3-12. doi:10.1089/brain.2011.0019

Satterthwaite, T. D., Wolf, D. H., Loughead, J., Ruparel, K., Elliott, M. A., Hakonarson, H., . . . Gur, R. E. (2012). Impact of in-scanner head motion on multiple measures of functional connectivity: Relevance for studies of neurodevelopment in youth. NeuroImage, 60, 623632. doi:10.1016/j.neuroimage.2011.12.063

Schweizer, K., \& Moosebrugger, H. (2004). Attention and working memory as predictors of intelligence. Intelligence, 32, 329-347. doi:10.1016/j.intell.2004.06.006

Seeley, W. W., Menon, V., Schatzberg, A. F., Keller, J., Glover, G. H., Kenna, H., . . Greicius, M. D. (2007). Dissociable intrinsic connectivity networks for salience processing and executive control. Journal of Neuroscience, 27, 2349-2356. doi:10.1523 /JNEUROSCI.5587-06.2007

Shapiro, S. L., Carlson, L. E., Astin, J. A., \& Freedman, B. (2006). Mechanisms of mindfulness. Journal of Clinical Psychology, 62, 373-386. doi:10.1002/jclp.20237

Sheehan, D. V., Lecrubier, Y., Sheehan, K. H., Amorim, P., Janavs, J., Weiller, E., . . . Dunbar, G. C. (1998). The Mini-International Neuropsychiatric Interview (M.I.N.I.): The development and validation of a structured diagnostic psychiatric interview for DSM-IV and ICD-10. Journal of Clinical Psychiatry, 59(Suppl. 20), 22-33.

Shucard, J. L., McCabe, D. C., \& Szymanski, H. (2008). An event-related potential study of attention deficits in posttraumatic stress disorder during auditory and visual $\mathrm{Go} / \mathrm{NoGo}$ continuous performance tasks. Biological Psychology, 79, 223-233. doi:10.1016/j. biopsycho.2008.05.005

Spencer, A. E., Faraone, S. V., Bogucki, O. E., Pope, A. L., Uchida, M., Milad, M. R., . . . Biederman J. (2016). Examining the association between posttraumatic stress disorder and attention-deficit/hyperactivity disorder: A systematic review and meta-analysis. Journal of Clinical Psychiatry, 77, 72-83. doi:10.4088/JCP.14r09479

Sripada, R., King, A., Garfinkel, S., Wang, X., Sripada, C., Welsh, R., \& Liberzon, I. (2012). Altered resting-state amygdala functional connectivity in men with posttraumatic stress disorder. Journal of Psychiatry \& Neuroscience, 37, 241-249. doi:10.1503/jpn.110069

Sripada, R. K., King, A. P., Welsh, R. C., Garfinkel, S. N., Wang, X., Sripada, C. S., \& Liberzon, I. (2012). Neural dysregulation in posttraumatic stress disorder: Evidence for disrupted equilibrium between salience and default mode brain networks. Psychosomatic Medicine, 74, 904-911. doi:10.1097/PSY.0b013e318273bf33

Stein, M. B., Kennedy, C. M., \& Twamley, E. W. (2002). Neuropsychological function in female victims of intimate partner violence with and without posttraumatic stress disorder. Biological Psychiatry, 52, 1079-1088. doi:10.1016/S0006-3223(02)01414-2

Steudte-Schmiedgen, S., Stalder, T., Kirschbaum, C., Weber, F., Hoyer, J., $\&$ Plessow, F. (2014). Trauma exposure is associated with increased context-dependent adjustments of cognitive control in patients with posttraumatic stress disorder and healthy controls. Cognitive, Affective, \& Behavioral Neuroscience, 14, 1310-1319. doi:10.3758/s13415-014-0299-2

Sutker, P. B., Vasterling, J. J., Brailey, K., \& Allain, A. N., Jr. (1995). Memory, attention, and executive deficits in POW survivors: Contributing biological and psychological factors. Neuropsychology, 9, 118-125.

Sylvester, C. M., Corbetta, M., Raichle, M. E., Rodebaugh, T. L., Schlaggar, B. L., Sheline, Y. I., . . Lenze, E. J. (2012). Functional network dysfunction in anxiety and anxiety disorders. Trends in Neurosciences, 35, 527-535. doi:10.1016/j.tins.2012.04.012

Thiel, C. M., Zilles, K., \& Fink, G. R. (2004). Cerebral correlates of alerting, orienting and reorienting of visuospatial attention: An event-related fMRI study. NeuroImage, 21, 318-328. doi:10.1016 /j.neuroimage.2003.08.044

Tursich, M., Ros, T., Frewen, P. A., Kluetsch, R. C., Calhoun, V. D., \& Lanius, R. A. (2015). Distinct intrinsic network connectivity patterns of post-traumatic stress disorder symptom clusters. Acta Psychiatrica Scandinavica, 132, 29-38. doi:10.1111/acps. 12387

Tzourio-Mazoyer, N., Landeau, B., Papathanassiou, D. Crivello, F. Etard, O. Delcroix, N., . . Joliot, M. (2002). Automated anatomical labeling of activations in SPM using a macroscopic anatomical parcellation of the MNI MRI single-subject brain. NeuroImage, 15, 273-289. doi:10.1006/nimg.2001.0978

van den Heuvel, M. P., Stam, C. J., Kahn, R. S., \& Hulshoff Pol, H. E. (2009). Efficiency of functional brain networks and intellectual performance. Journal of Neuroscience, 29, 7619-7624. doi:10.1523 /JNEUROSCI.1443-09.2009

van Donkelaar, P., Langan, J., Rodriguez, E., Drew, A., Halterman, C., Osternig, L. R., . . Chou, L. (2005). Attentional deficits in concussion. Brain Injury, 19, 1031-1039. doi:10.1080 /02699050500110363

Vasterling, J. J., Duke, L. M., Brailey, K., Constans, J. I., Allain, A. N., \& Sutker, P. B. (2002). Attention, learning, and memory performances and intellectual resources in Vietnam veterans: PTSD and no disorder comparisons. Neuropsychology, 16, 5-14. doi:10.1037/08944105.16.1.5 
Vossel, S., Weidner, R., Driver, J., Friston, K. J., \& Fink, G. R. (2012). Deconstructing the architecture of dorsal and ventral attention systems with dynamic causal modeling. Journal of Neuroscience, 32, 10637-10648. doi:10.1523/JNEUROSCI.0414-12.2012

Weierich, M. R., Treat, T. A., \& Hollingworth, A. (2008). Theories and measurement of visual attentional processing in anxiety. Cognition and Emotion, 22, 985-1018. doi:10.1080/02699930701597601

Westlye, L. T., Grydeland, H., Walhovd, K. B., \& Fjell, A. M. (2011). Associations between regional cortical thickness and attentional networks as measured by the attention network test. Cerebral Cortex, 21, 345-356. doi:10.1093/cercor/bhq101

Winship, C., \& Korenman, S. (1997). Does staying in school make you smarter? The effect of education on IQ in The bell curve. In B. Devlin, S. E. Fienberg, D. P. Resnick, \& K. Roeder (Eds.), Intelligence, genes, and success: Scientists respond to The Bell Curve (pp. 215-234). New York, NY: Springer. Retrieved from http://link.springer.com/chapter/10.1007/978-1-4612-0669-9_10

Yeo, B. T. T., Krienen, F. M., Sepulcre, J., Sabuncu, M. R., Lashkari, D., Hollinshead, M., . . Buckner, R. L. (2011). The organization of the human cerebral cortex estimated by intrinsic functional connectivity.
Journal of Neurophysiology, 106, 1125-65. doi:10.1152 jjn.00338.2011

Yin, Y., Jin, C., Eyler, L. T., Jin, H., Hu, X., \& Duan, L. (2012). Altered regional homogeneity in post-traumatic stress disorder: a restingstate functional magnetic resonance imaging study. Neuroscience Bulletin, 28, 541-549. doi:10.1007/s12264-012-1261-3

Zhang, Y., Liu, F., Chen, H., Li, M., Duan, X., Xie, B., \& Chen, H. (2015). Intranetwork and internetwork functional connectivity alterations in post-traumatic stress disorder. Journal of Affective Disorders, 187, 114-121. doi:10.1016/j.jad.2015.08.043

Zhang, Y., Xie, B., Chen, H., Li, M., Liu, F., \& Chen, H. (2016). Abnormal functional connectivity density in post-traumatic stress disorder. Brain Topography, 29, 405-411. doi:10.1007/s10548016-0472-8

Zhou, Y., Wang, Z., Qin, L., Wan, J., Sun, Y., Su, S., . . Xu, J. (2012). Early altered resting-state functional connectivity predicts the severity of post-traumatic stress disorder symptoms in acutely traumatized subjects. PLoS ONE, 7, e46833. doi:10.1371/journal. pone. 0046833 